\title{
Microvascular Myocutaneous and Cutaneous Free Flap Reconstruction for Patients With Terminal Esophagostomy After Complicated Oncological Esophagus Resection
}

\section{Lukas Poelsler}

Paracelsus Medical University

Oliver Koch ( $D$ o.koch@salk.at )

Paracelsus Medical University

\section{Alexander Gaggl}

Paracelsus Medical University

Jaroslav Presl

Paracelsus Medical University

Jörg Hutter

Paracelsus Medical University

Christian Brandtner

Paracelsus Medical University

Klaus Emmanuel

Paracelsus Medical University

\section{Research Article}

Keywords: esophageal cancer, free flaps, anastomotic leak, cervical esophagostomy, surgical oncology

Posted Date: July 20th, 2021

DOI: https://doi.org/10.21203/rs.3.rs-717866/v1

License: (c) (i) This work is licensed under a Creative Commons Attribution 4.0 International License.

Read Full License

Version of Record: A version of this preprint was published at European Surgery on December 23rd, 2021. See the published version at https://doi.org/10.1007/s10353-021-00744-6. 


\section{Abstract}

\section{Background}

Persisting anastomotic leakage after oncological esophagectomy is a severe problem associated with high mortality and morbidity. Unfortunately, treatment options with promising results are scarce especially when conventional operative and endoscopic methods have failed. Due to limitation of oral intake and need for artificial nutrition quality of life is reduced. Microvascular myocutaneous and cutaneous free flap (MFF) reconstruction could be a promising alternative.

\section{Methods}

This retrospective case series presents seven patients treated between March 2017 and November 2020 at our surgical department, with persisting postoperative anastomotic leakage without further feasible treatment options. All patients received anastomotic MFF reconstruction, we evaluated used flaps and important intraoperative factors like microvascular anastomosis. Complications were assessed by Clavien-Dindo-Score, patient contentment was evaluated with a questionnaire.

\section{Results}

The included seven male patients had median age of 65.15 years (range: 48-75). MFF function was adequate in six out of seven patients, one flap necrosis was encountered. Five patients initially had good results, surgical revision was performed in one patient to ensure graft function. Postoperative complications appeared in 6 out of 7 patients, three patients had complications ranked in Clavien-Dindo IIla (two patients received stenting, one patient endoscopic stenting and dilatation) and two patients needed surgical revision (Clavien-Dindo IIIb). Furthermore one patient had postoperative delirium with prolonged intensive care unit stay and one patient had no complications (Clavien-Dindo 0). Mean duration of inpatient care was 63 days (Range: 24-156). At time of evaluation, one patient has died because of his malignant disease. No more additional nutrition was needed in 3 out of 6 patients with adequate graft function. The majority of patients reported improved quality of life after MFF.

\section{Conclusion}

MFF free flap can be an alternative treatment option for patients with terminal esophagostomy after complicated oncological esophagus resection without further treatment options. The renewed ability of oral food intake results in a significant improvement of quality of life for the patients.

\section{Background}

Esophageal cancer remains a rare diagnosis with limited prognosis in the western world. In Austria 423 patients were diagnosed with esophagus cancer in 2018. [1] Squamous cell carcinoma (SCC) and adenocarcinoma are the most frequently observed types of esophageal cancer with 5.2 per 100.000 for SCC and 0.7 per 100.000 for adenocarcinoma. SCC is most frequently observed in Asia whereas 
adenocarcinoma is more frequently observed in Europe, with rising incidence compared to SCC in recent years. [2] Medication based therapies for oncological diseases have rapidly improved over the years. [3] However, surgical esophagus resection is considered as gold standard treatment and as only potentially curative treatment option. Locally advanced tumors should receive neoadjuvant therapy to lower regiolocal recurrence rates and to ensure $\mathrm{R} 0$ resection. Widely used treatment options are neoadjuvant chemotherapies as well as neoadjuvant chemoradiation. In Europe currently the most commonly used neoadjuvant chemoradiation scheme is CROSS. Preoperative radiation is known to be an independent risk factor for local complications, complications appear more often especially when radiation dosage increases. [4-6]

Typically, esophagus resection is followed by gastric tube pull-up reconstruction, either anatomical or extraanatomical retrosternal pull-up. SCC usually requires McKeown esophagectomy with cervical anastomosis, and adenocarcinoma can more often be treated by Ivor-Lewis esophagectomy with intrathoracic anastomosis. Minimal invasive operation techniques have improved patient outcome lately, yet esophagus resection remains a procedure with high mortality and morbidity. Especially anastomosis insufficiency remains a major problem. In patients with SCC who received cervical anastomosis prognosis depends on limitation to cervical region or further intrathoracic manifestation if insufficiency appears. Thoracic manifestation is associated with higher mortality and morbidity. Major reason for persisting anastomotic leakage is gastric pull-up tube necrosis. This complication appears scares, approximately $1 \%$ of esophagus resections with gastric pull-up lead to necrosis of gastric conduit with need for surgical revision. [7] Further used reconstruction techniques are colonic interposition or interposition of small bowel. Colonic interposition requires more anastomosis, which increases risk of anastomotic leak. Therefore, it is less frequently used. [8] However, treatment options are scarce if secondary interposition develops necrosis as well or fistulation occurs and sometimes terminal esophagostomy is the only solution. $[9,10]$ This condition is associated with poor quality of life due to impossibility of oral intake. Quality of life is most impacted by possibility of oral nutrition, even in jejunum interposition quality of life is described to improve significantly. [11] Need for in-hospital-stay is frequent and therefore associated with high costs.

Despite multiple reconstructive approaches proposed in the past, there remains no consensus for the optimal method of reconstructing a terminal esophagostomy. $[5,10,12,13]$. Especially in patients after complicated postoperative course who received preoperative radiotherapy and are without treatment options. Microvascular myocutaneous and cutaneous free flap reconstruction could be a promising alternative for such patients. The use for esophageal reconstruction has already been described previously. [13] Free flap reconstruction has also been described as successful technique for reconstruction of complex mediastinal tracheal defects. [14] The aim of this paper is to analyze the outcome of microvascular myocutaneous and cutaneous free flap reconstruction for patients with terminal esophagostomy respectively persisting cervical anastomotic leak after complicated oncological esophagus resection for SCC at our surgical department. 


\section{Patients And Methods}

We performed a retrospective analysis of all patients who received MFF reconstruction between March 2017 and November 2020 at University Hospital Salzburg in cooperation of Department of Surgery and Department of Oral and Maxillofacial Surgery.

The protocol for the research project has been approved by Ethics Committee of the institution within which the work was undertaken, and it conforms to the provisions of the Declaration of Helsinki in 1995 (as revised in Edinburgh 2000). Informed consent was obtained from all subjects. The research project was undergone without any financial assistance and there are no relationships that may pose conflict of interest.

In the study period 7 patients were treated. All patients who received MFF reconstruction were male. Six patients initially received oncological esophagus resection because of SCC, one patient initially received hypopharyngeal resection after SCC diagnosis followed by esophageal resection because of esophageal recurrence. Gastric pull-up was performed in all seven patients. Secondary carcinoma was diagnosed in three out of seven patients (42.8\%) at staging procedure with hypopharynx being the most common location. Postoperatively all patients developed severe complications leading salivary stomata operation. Endoscopic therapies like Endo-VAC, endoscopic dilatation and continuous endoscopic monitoring over short period of time was performed. Additionally four patients received colon interposition. Furthermore, one patient received jejunum interposition after developing necrosis of colonic interposition. Endoscopic and open vacuum-pressure therapy (VAC) was performed in six patients (85.7\%). Indication for primary MFF replacement after gastric pull-up necrosis was imposed in two patients because of tracheal fistulation.

Mean age of the patients was 65.15 years (range: $48-75$ years). Mean preoperative weight was $59.86 \mathrm{~kg}$ (range: $49-74 \mathrm{~kg}$ ) with mean size of $175.14 \mathrm{~cm}$ (range: 160-183 kg). Body-Mass-Index (BMI) was evaluated, mean BMI was $19.45 \mathrm{~kg} / \mathrm{m}^{2}$ (range: $16-23.36 \mathrm{~kg} / \mathrm{m}^{2}$ ). Six out of seven patients $(85.7 \%)$ received neoadjuvant radiation before resection of primary. Gray doses was available in 4 out of 6 patients (66.7\%). Mean Gray dosage was 58.075 Gray (range: 41.4-66.0 Gray). TNM-LVR classification, Grading and UICC stadium is reported in Table 1. 
Table 1

TNM-LVR classification, UICC-Stadium and Grading of primary tumors

\begin{tabular}{|c|c|c|}
\hline Classifications & Number of patients & $\%$ \\
\hline \multicolumn{3}{|l|}{ T-Stadium } \\
\hline Tis & 1 & 14,3 \\
\hline $\mathrm{T} 1 \mathrm{~b}$ & 1 & 14,3 \\
\hline $\mathrm{T} 2$ & 1 & 14,3 \\
\hline T3 & 3 & 42,8 \\
\hline \multicolumn{3}{|l|}{ N-Stadium } \\
\hline No & 4 & 57,1 \\
\hline N1 & 2 & 28,6 \\
\hline \multicolumn{3}{|l|}{ M Stadium } \\
\hline MO & 6 & 85,7 \\
\hline \multicolumn{3}{|l|}{ L-Stadium } \\
\hline LO & 6 & 85,7 \\
\hline \multicolumn{3}{|l|}{ V-Stadium } \\
\hline V0 & 6 & 85,7 \\
\hline \multicolumn{3}{|l|}{ R-Stadium } \\
\hline RO & 5 & 71,4 \\
\hline R1 & 1 & 14,3 \\
\hline \multicolumn{3}{|c|}{ Stage grouping according to UICC } \\
\hline 0 & 1 & 14,3 \\
\hline IA & 1 & 14,3 \\
\hline IB & 1 & 14,3 \\
\hline IIA & 1 & 14,3 \\
\hline IIIA & 2 & 28,6 \\
\hline \multicolumn{3}{|l|}{ Grading } \\
\hline G2 & 3 & 42,8 \\
\hline G3 & 2 & 28,6 \\
\hline
\end{tabular}


T3 stadium was most frequently observed. Complete data was available in 6 out of 7 patients. All patients received reconstruction with microvascular myocutaneous and cutaneous free flap, the used free flaps are reported in Table 2.

Table 2

Overview of used free flaps

\begin{tabular}{|ll|}
\hline Type of microvascular myocutaneous and cutaneous free flap & Number of uses (\%) \\
\hline Anterior lateral thigh free flap (ALT) & $4(57.1 \%)$ \\
\hline Radialis free flap & $1(14.3 \%)$ \\
\hline Osteomyocutaneous free flap from medial femoral condyle & $1(14.3 \%)$ \\
\hline Left brachial free flap & $1(14.3 \%)$ \\
\hline
\end{tabular}

The flaps were used to restore continuity of oral cavity and esophagus. This was achieved by harvesting the flap at first with sufficient length of appertain vessels (e.g. Figure 1.1, 1.2, 1.4, 2.1). After a successful checking for sufficient perfusion, the flap was transferred to the neck (e.g. Figure 1.3, 2.3, 2.4.). The flap was disposed in a circular form (e.g. Figure 1.3,2.2) and then connected to gastric conduit or colonic interposition.

The used vessels for microvascular anastomosis are reported in Table 3.

Table 3

Used vessels for microvascular anastomosis

\begin{tabular}{|ll|}
\hline Vessel for microvascular anastomosis & Number of uses (\%) \\
\hline Arteria thyroidea superior & $5(71.4 \%)$ \\
\hline Arteria facialis & $1(14.3 \%)$ \\
\hline Arteria transversa colli & $1(14.3 \%)$ \\
\hline
\end{tabular}

\section{Complications and patient contentment}

Complications were obtained according to Clavien-Dindo Classification. Additionally, we evaluated patient contentment after surgical procedure with three questions ("Are you able to swallow appropriately?" "Did your Quality of Life improve, stay steady or worsen since operation was performed?" "With the knowledge now, would you consent in this procedure again?"). 


\section{Results}

Graft results

MFF function was adequate in six out of seven patients (85.7\%); flap necrosis appeared in one patient (14.3\%). Flap results were initially good in five patients, one more patient needed surgical revision to ensure graft function. No local complications appeared after free flap mobilization. Speaking was not feasible in both patients with tracheal fistulation, one patient regained speaking ability, one patient can speak with appropriate tracheal canula. Patient with osteomyocutaneous free flap from medial femoral condyle developed satisfactory result: no complications were obtained and full ability of speaking was regained by the patient. Lateral-arm-flap was rejected, yet this graft choice can be applicated.

Complications and patient contentment

Of six patients four reported improved quality of life, results of the three questions are reported in Table 4. No more additional artificial nutrition was needed in three out of six patients $(50 \%)$. Five out of six patients would consent in this procedure again. Postoperative complications appeared in six out of seven patients, three patients had complications ranked in Clavien-Dindo Illa (two patients received stenting, one patient endoscopic stenting) and two patients needed surgical revision (Clavien-Dindo IIIb). Furthermore, one patient had postoperative delirium with prolonged intensive care unit stay and one patient had no complications (Clavien-Dindo 0). Mean duration of inpatient care was 63 days (Range: 24-156). At time of evaluation, one patient has died because of his malignant disease.

Table 4

Three questions to assess patient contentment

\begin{tabular}{|c|c|c|}
\hline Asked questions & Possible answers & $\begin{array}{l}\text { Number of } \\
\text { answers }(n= \\
6)\end{array}$ \\
\hline \multirow[t]{3}{*}{ Question 1: Are you able to swallow appropriately? } & Yes, even solid food & $3(50 \%)$ \\
\hline & $\begin{array}{l}\text { Yes, but with } \\
\text { cutaneous } \\
\text { fistulation }\end{array}$ & $1(16.7 \%)$ \\
\hline & No & $2(33.3 \%)$ \\
\hline \multirow[t]{4}{*}{$\begin{array}{l}\text { Question 2: Did your Quality of Life improve, stay steady or } \\
\text { worsen since operation was performed? }\end{array}$} & $\begin{array}{l}\text { Massive } \\
\text { improvement }\end{array}$ & $2(33.3 \%)$ \\
\hline & improvement & $1(16.7 \%)$ \\
\hline & steady & $2(33.3 \%)$ \\
\hline & worsen & $1(16.7 \%)$ \\
\hline \multirow{2}{*}{$\begin{array}{l}\text { With the knowledge now, would you consent in this } \\
\text { procedure again? }\end{array}$} & Yes & $5(83.3 \%)$ \\
\hline & No & $1(16.7 \%)$ \\
\hline
\end{tabular}




\section{Discussion}

The results of this paper shows that MFF free flap is a safe and feasible treatment option for patients with terminal esophagostomy after complicated oncological esophagus resection. There was no procedure related mortality observed and only one patient reported worsening of quality of life while patients with sufficient graft function reported massive regain of quality of life. Therefore, especially due to high subjective level of suffering and low quality of life this procedure can be considered. Despite the occurred complications and insufficient results in some patients, all patients besides one would consent into this treatment option again. That implicates the subjective poor quality of life prior to surgery. Number of patients who regained the ability of oral food intake was encouraging, especially in consideration of few other available treatment option with permanent need for cervical esophagostomy. Adequate graft function resulted in a significant improvement of quality of life for the patients. None patient reported lower quality of life than before free flap reconstruction. Anterior lateral thigh (ALT) free flap reconstruction is a frequently used free flap reconstruction technique for head and neck reconstructions, since its initial description in 1983. [15] However, free flaps with vascularized bone components are described to deliver better results due to a lower risk of reabsorption. Free flap harvesting from the medial knee are performed since the early 1990s and can be used for reconstruction of postoperative defects, especially after tumor resection. However, vascular anatomy may diverge. Therefore, routinely preoperative vascular imaging is required before flap harvesting. [16, 17]. Radial forearm free flaps are also commonly used for reconstructions in the head and neck area. Still, free flap failure is a scarce but occurring problem. Graft failure is differentiated in early and late graft failure, with failure after seven postoperative day of surgery considered as late failure. The most important risk factor for graft failure is preoperative irradiation. Most frequent observed reasons for late graft failure are abscess formation and vascular compromise. Careful graft observation is recommended within the first 14 postoperative days to detect graft dysfunction early. $[18,19]$ Unfortunately, one graft was initially rejected in our group. Due to small size of total sample, some grafts were only used once. Result of graft does therefore not implicate about general outcome. Flaps for reconstruction should be selected individually and mutually with the patient. Rejection of graft remains a rare but occurring problem with few possible preventions. Treatment options are ending in patients with multiple failed reconstruction techniques after esophagus resection. Therefore, every further applied strategy needs to be well considered and discussed with the patient. MFF reconstruction offers an additional treatment option for those patients. However, this procedure has high-risks with numerous possible complications. Patients eligible for this treatment should be selected carefully and perioperative risks have to be reduced preoperatively for example by adequate monitoring of diabetes and sufficient hemoglobin levels.

This study has potential limitations. We performed MFF reconstruction on a rather small patient sample. Furthermore, this presentation is retrospective which is known for limitations like in this case the analysis of quality of life and available data of patients treated extramurally in detailed demographic data and primary tumor stadium. 
However, the outcome of the patients after MFF reconstruction in this paper shows that the method might be a relevant treatment option to be discussed with patients if expertise in free flap reconstruction and esophageal surgery is available at primary treatment center.

In conclusion MFF reconstruction can be an alternative treatment option in patients with complicated postoperative courses with failing of primary reconstruction. MFF reconstruction has its role in patients with special defects like tracheal fistulation or as option after conventional strategies failed. Due to ending therapy strategies newly applied strategies need to be discussed in detail with all beneficial and possible adverse aspects with the patient. For patients without further feasible treatment options MFF reconstruction is a promising alternative to improve quality of life.

\section{Declarations}

\section{Conflict of interest}

The authors declare that there are no conflict of interests.

\section{Author contributions}

All authors contributed to the study conception and design. Material preparation, data collection and analysis were performed by Lukas Poelsler, Oliver Koch and Klaus Emmanuel. The first draft of the manuscript was written by Lukas Poelsler and all authors commented on previous versions of the manuscript. All authors read and approved the final manuscript.

\section{References}

1. Österreichisches Krebsregister und Todesursachenstatistik [Internet]. Statistik Austria. 2020 [cited 14.02.2021].

2. Arnold M, Soerjomataram I, Ferlay J, et al. Global incidence of oesophageal cancer by histological subtype in 2012. Gut. 2015;64(3):381-7.

3. Fatehi Hassanabad A, Chehade R, Breadner D, et al. Esophageal carcinoma: Towards targeted therapies. Cell Oncol (Dordr). 2020;43(2):195-209.

4. Bang A, Broomfield JA, Chan J, et al. Radiation dose mapping and anastomotic complications after trimodality therapy for esophageal cancers. Clin TransI Radiat Oncol. 2019;15:76-82.

5. Lordick F, Mariette C, Haustermans K, et al. Oesophageal cancer: ESMO Clinical Practice Guidelines for diagnosis, treatment and follow-up. Ann Oncol. 2016;27(suppl 5):v50-v7.

6. van Hagen P, Hulshof MC, van Lanschot JJ, et al. Preoperative chemoradiotherapy for esophageal or junctional cancer. N Engl J Med. 2012;366(22):2074-84.

7. Low DE, Kuppusamy MK, Alderson D, et al. Benchmarking Complications Associated with Esophagectomy. Ann Surg. 2019;269(2):291-8. 
8. Duan X, Bai W, Ma Z, et al. Management and outcomes of anastomotic leakage after McKeown esophagectomy: A retrospective analysis of 749 consecutive patients with esophageal cancer. Surg Oncol. 2020;34:304-9.

9. Fearon NM, Mohan HM, Fanning M, et al. Colonic interposition, a contemporary experience: technical aspects and outcomes. Updates Surg. 2020.

10. Marks JL, Hofstetter WL. Esophageal reconstruction with alternative conduits. Surg Clin North Am. 2012;92(5):1287-97.

11. Baker CR, Forshaw MJ, Gossage JA, et al. Long-term outcome and quality of life after supercharged jejunal interposition for oesophageal replacement. Surgeon. 2015;13(4):187-93.

12. Markar S, Gronnier C, Duhamel A, et al. Salvage Surgery After Chemoradiotherapy in the Management of Esophageal Cancer: Is It a Viable Therapeutic Option? J Clin Oncol. 2015;33(33):3866-73.

13. Sokoya M, Vincent A, Cohn JE, et al. Comparison of radial forearm free flap and gastric pull-up in pharyngo-oesophageal reconstruction. Clin Otolaryngol. 2019;44(3):405-7.

14. Maitani K, Yamasaki M, Otani N, et al. Successful reconstruction of an intrathoracic tracheal defect using a muscle flap and conchal cartilage graft. Esophagus. 2021;18(2):416-9.

15. Baek SM. Two new cutaneous free flaps: the medial and lateral thigh flaps. Plast Reconstr Surg. 1983;71(3):354-65.

16. Chen YB, Chen HC, Hahn LH. Major mandibular reconstruction with vascularized bone grafts: indications and selection of donor tissue. Microsurgery. 1994;15(4):227-37.

17. Weitgasser $L$, Cotofana $S$, Winkler $M$, et al. Detailed vascular anatomy of the medial femoral condyle and the significance of its use as a free flap. J Plast Reconstr Aesthet Surg. 2016;69(12):1683-9.

18. Forner D, Williams BA, Makki FM, et al. Late free flap failure in head and neck reconstruction: A systematic review. Ear Nose Throat J. 2018;97(7):213-6.

19. Zhou W, Zhang WB, Yu Y, et al. Risk factors for free flap failure: a retrospective analysis of 881 free flaps for head and neck defect reconstruction. Int J Oral Maxillofac Surg. 2017;46(8):941-5.

\section{Figures}




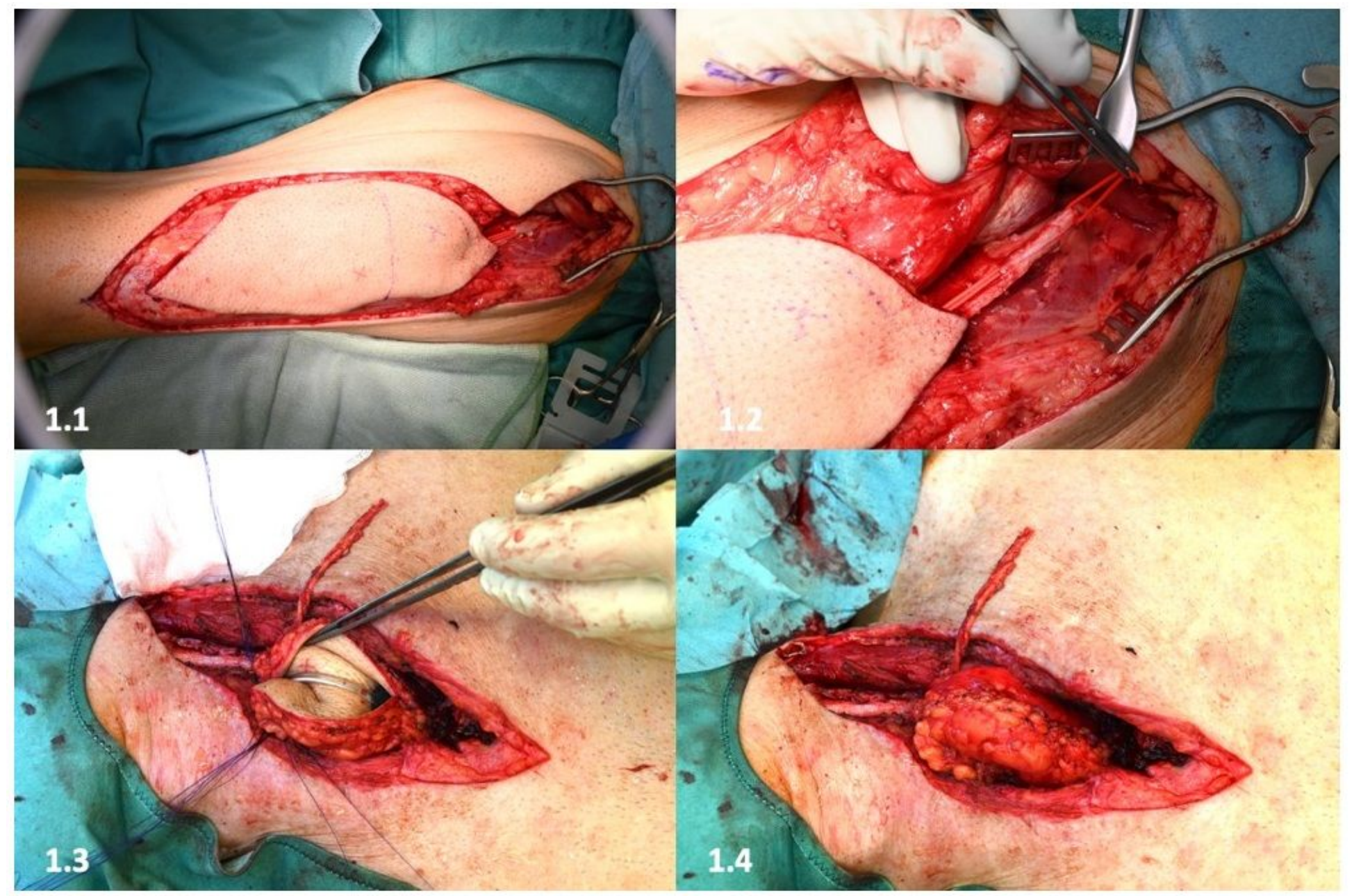

\section{Figure 1}

Intraoperative pictures of ALT reconstruction (1.1 harvesting of ALT free flap, 1.2. vessel preparation, 1.3. placement of ALT as interposition to ensure esophagus continuity with placed gastric tube, 1.4. finished placement of ALT with newly functionating esophagus) 


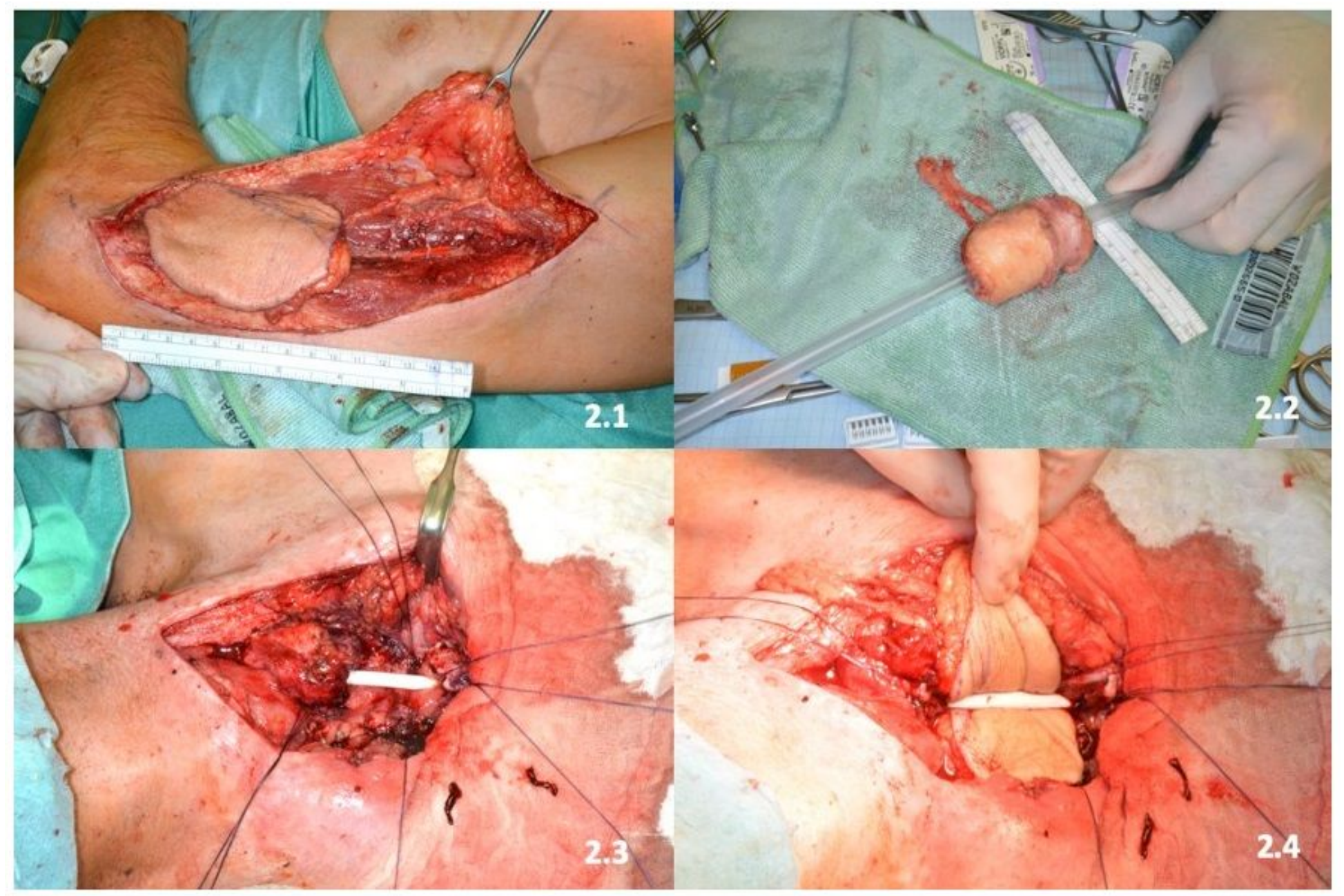

Figure 2

Intraoperative pictures of lateral brachial free flap (2.1. harvesting of free flap, 2.2. circular formation of free flap, 2.3. preparations for free flap interposition, 2.4. placement of circular free flap with gastric tube) 